PoS $\quad \begin{aligned} & \text { PROCEEDINGS } \\ & \text { OF SCIENCE }\end{aligned}$

\title{
Searches for non-Standard Model decays to two light bosons of the Higgs boson
}

\section{Ariel Schwartzman, on behalf of the ATLAS Collaboration*}

SLAC National Accelerator Laboratory

E-mail: scheslac.stanford.edu

Theories beyond the Standard Model predict Higgs boson decays that do not exist in the Standard Model, such as decays into two light bosons $(a)$. This talk presents recent results based on pp collision data collected at $13 \mathrm{TeV}$ with the ATLAS detector at the LHC.

The 39th International Conference on High Energy Physics (ICHEP2018)

4-11 July, 2018

Seoul, Korea

${ }^{*}$ Speaker. 
Exotic Higgs boson decays are a powerful probe for physics beyond the Standard Model (SM). In particular, the very narrow Higgs decay width makes it sensitive to small couplings to non-SM particles. Current measurements at the LHC constrain non-SM branching ratio (BR) of the Higgs boson to less than $30 \%$ at $95 \% \mathrm{CL}$. This means that there is ample room for exotic Higgs boson decays compatible with observations to date, motivating direct searches for non-SM Higgs boson decays. Four different topologies for the decay of the Higgs boson to a pair of spin-zero particles $a$ or spin- $1 Z_{d}$ decaying into a pair of SM particles are discussed: $(b b)(b b),(b b)(\mu \mu),(l l)(l l)$, and $(\gamma \gamma)(g g)$. Data were recorded in 2015-2016 by the ATLAS detector [1] at the LHC, corresponding to an integrated luminosity of $36.1 \mathrm{fb}^{-1}$. The main experimental handles for the reconstruction of $\mathrm{H} \rightarrow a a$ events are the mass constrains that arise from the fact that the two $a$-bosons have the same mass and, combined, have the mass of the Higgs boson. There are two key experimental challenges. First, $a$-boson decay products tend to be soft, leading to physics objects often below the reconstruction threshold. Second, for light $a$-boson decays, the Lorentz boost can be large enough to lead to opening angles smaller than the angular size of physics objects. This means jets and leptons overlap and standard reconstruction techniques become inefficient, limiting the discovery reach at low mass.

\section{1. $\mathbf{H} \rightarrow a a \rightarrow 4 b$}

The search in the $4 b$ final state considers Higgs bosons produced in association with a $\mathrm{W}$ or a $\mathrm{Z}$ boson, and $a$-boson decays into b-quarks promptly or with $c \tau_{a}$ up to $6 \mathrm{~mm}$ [2]. Events are categorized according to the number of leptons, jets, and b-jets. Control regions (CR) are defined according to the two main background processes: $t \bar{t}+$ heavy flavour (HF) jets in the single lepton channel, and Z+HF jets in the dilepton channel. A boosted decision tree is trained in each signal region (SR) and for three $a$-boson masses. Dominant uncertainties arise from heavy flavour tagging and background and signal modeling. Combined observed upper limits for prompt decay vary between 3.0 and $1.3 \mathrm{pb}$ in the range of $m_{a}$ between 20 and $60 \mathrm{GeV}$. The best limits for long lived $a$-bosons range from 1.8 to $0.68 \mathrm{pb}$ for $c \tau_{a} \sim 0.4 \mathrm{~mm}$ [2].

\section{2. $\mathbf{H} \rightarrow a a \rightarrow b b \mu \mu$}

The presence of a muon pair in the final state provides a powerful signature for triggering and improved mass reconstruction. It also provides sensitivity to models with enhanced lepton couplings. The key experimental handle for this search consists of exploiting the fact that the dimuon invariant mass resolution $\left(m_{\mu \mu}\right)$ is ten times better than the invariant mass resolution from the b-jet pair $\left(m_{b b}\right)$. A kinematic fit, exploiting the symmetry of the $\mathrm{H} \rightarrow a a$ decays, leads to a factor of two improvement in the combined $m_{b b \mu \mu}$ resolution, as shown in Figure 2. The signal region requires $\left|m_{b b \mu \mu}-m_{H}\right|<15 \mathrm{GeV}$ and missing $E_{\mathrm{T}}$ less than $60 \mathrm{GeV}$. The sidebands of the $m_{b b \mu \mu}$ distribution are used as the Drell-Yan (DY) CR. A top CR is defined by inverting the upper bound on missing $E_{\mathrm{T}}$. The top background, estimated from simulation, and Drell-Yan, estimated from 0-tag data templates, are normalized in a profile likelihood fit to the data over the control and signal regions. The dominant uncertainties are due to the jet energy scale and resolution (JES/JER), signal 
and background modeling, and the DY template. The upper limit at 95\% CL on $\left(\frac{\sigma_{H}}{\sigma_{S M}}\right) \times B(H \rightarrow$ $a a \rightarrow b b \mu \mu)$ ranges between $10^{-4}$ and $10^{-3}$ for $20<m_{a}<60 \mathrm{GeV} \mathrm{[3].}$
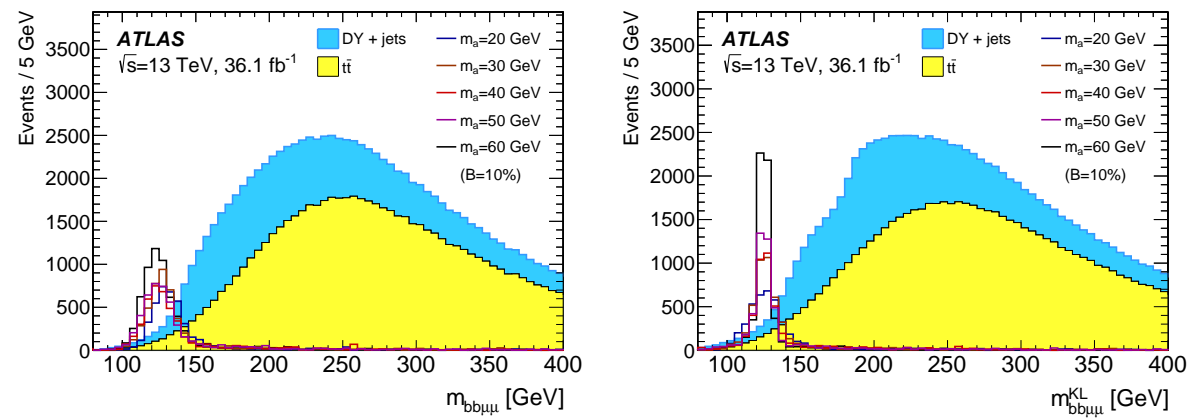

Figure 1: The (left) $m_{b b \mu \mu}$ before and (right) after the KL fit for events after the preselection stage, but removing the upper bound on $m_{\mu \mu}$ [3]. The $t \bar{t}$ contribution is modelled with the simulated sample normalised to the theoretical cross-section. The Drell-Yan contribution is taken from data templates and normalised to the total yield predicted by the Drell-Yan simulation. The signal distributions for all five simulated $m_{a}$ are also shown assuming the SM Higgs boson cross-section (including ggF, VBF and VH production) and $\mathrm{B}(\mathrm{H} \rightarrow a a \rightarrow b b \mu \mu)=10 \%$ [3].

\section{3. $\mathbf{V B F ~} \mathrm{H} \rightarrow a a \rightarrow \gamma \gamma g g$}

A search for $a$-bosons decaying to a pair of photons and a pair of gluons provides sensitivity to models where fermionic decays are suppressed and the $a$-boson can only decay to photons or gluons. The Vector Boson Fusion (VBF) production mode is utilized as it provides higher cross section than $\mathrm{VH}$ and provides additional experimental handles to suppress backgrounds. The analysis uses a di-photon trigger, and requires four or more jets, two of which satisfy a VBF selection with $m_{j j}^{\mathrm{VBF}}>500 \mathrm{GeV}$ and leading jet $p_{\mathrm{T}}>60 \mathrm{GeV}$. Forward pileup jets, outside the $\eta$ coverage of the tracker, constitute a major experimental challenge for this final state because standard track-based pileup jet suppression algorithms are not available. This analysis makes use of a novel forward pileup jet suppression method: forward jet vertex tagging (fJVT) [6]. The key idea of this method is that jets produced in pileup interactions are balanced in $p_{\mathrm{T}}$. Therefore, forward pileup jets can be identified by looking for a back-to-back jet in the central region for which tracking information is available. Thus, fJVT uses tracks in the central region to indirectly tag forward pileup jets. This new technique, as shown in Figure 3, significantly reduces the contamination from pileup jets, improving the VBF signal purity up to a factor of 4 for events with an average pileup of 35 . The analysis uses a data driven ABCD background estimation method based on photon isolation and $\left|m_{j j}-m_{\gamma \gamma}\right|$. The dominant uncertainties are due to the low number of events, and JES/JER. Upper limits on $\left(\frac{\sigma_{H}}{\sigma_{S M}}\right) \times B(H \rightarrow a a \rightarrow \gamma \gamma g g)$ range between 0.06 and 0.16 for $20<m_{a}<60 \mathrm{GeV}$ [5]. This search is complementary to $H \rightarrow a a \rightarrow \gamma \gamma \gamma \gamma$ and more sensitive to scenarios with enhanced gluon couplings.

\section{Summary}

Exotic Higgs boson decays provide a window to search for physics beyond the Standard 

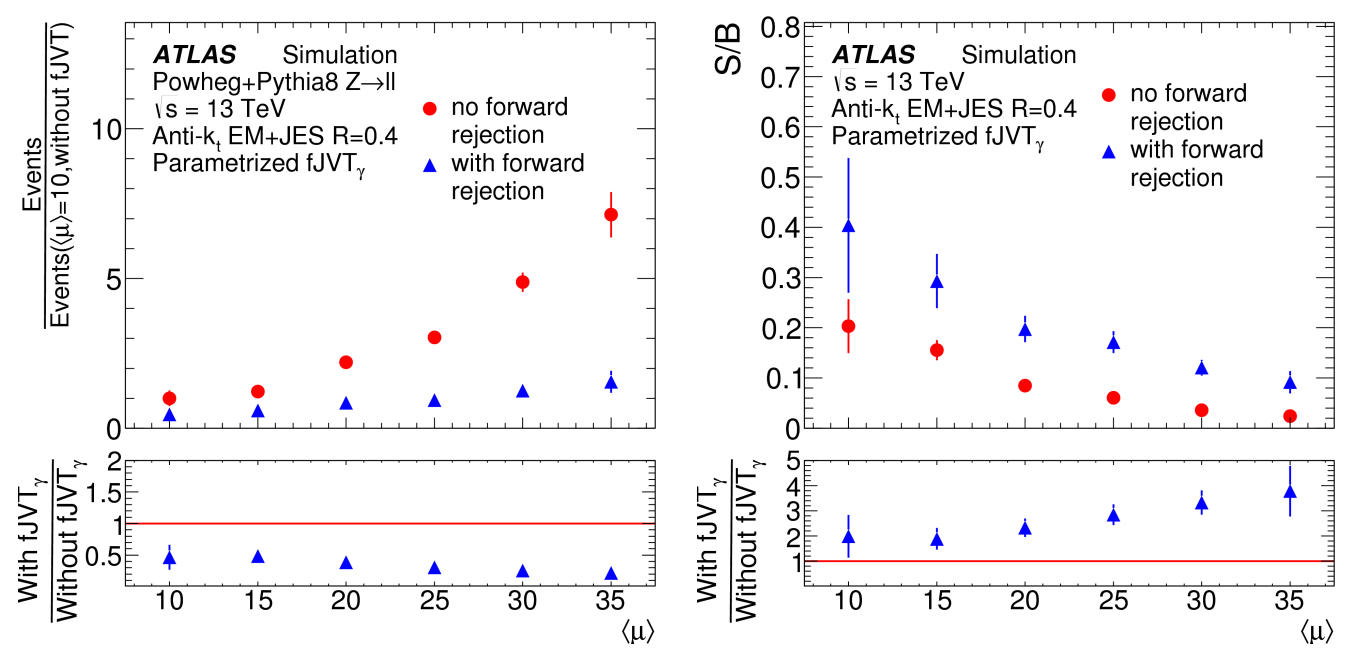

Figure 2: Relative expected yield variation of $Z \rightarrow l l$ events (Left) and VBF $H \rightarrow \tau \tau$ signal purity (Right) as a function of the number of interactions per bunch crossing $(\langle\mu\rangle)$. The expected signal and background yields at $\langle\mu\rangle=10$ are used as reference. Parameterized hard-scatter efficiency and pile-up efficiency are used. The lower panels display the ratio to the reference without pile-up rejection [6].

Model. ATLAS has performed several searches for exotic decays of the Higgs boson to two light bosons using $36.1 \mathrm{fb}^{-1}$ of data in multiple final states. Data has been found consistent with SM background predictions, leading to upper limits on the branching ratio for non-SM decays of the Higgs boson to two light bosons. The next steps for these searches is to enhance the discovery potential to low $m_{a}$ where the decay products of the $a$-boson merge and current reconstruction techniques are not suitable.

\section{References}

[1] ATLAS Collaboration, The ATLAS Experiment at the CERN Large Hadron Collider, 2008 JINST 3 S08003.

[2] ATLAS Collaboration, Search for the Higgs boson produced in association with a vector boson and decaying into two spin-zero particles in the $\mathrm{H} \rightarrow \mathrm{aa} \rightarrow 4 \mathrm{~b}$ channel in pp collisions at $\sqrt{s}=13 \mathrm{TeV}$ with the ATLAS detector, JHEP 10 (2018) 031, arXiv:1806.07355.

[3] ATLAS Collaboration, Search for Higgs boson decays into a pair of light bosons in the bb $\mu \mu$ final state in pp collision at $\sqrt{s}=13 \mathrm{TeV}$ with the ATLAS detector, CERN-EP-2018-153, arXiv:1807.00539.

[4] ATLAS Collaboration, Search for Higgs boson decays to beyond-the-Standard-Model light bosons in four-lepton events with the ATLAS detector at $\sqrt{s}=13 \mathrm{TeV}$, JHEP 06 (2018) 166, arXiv:1802.03388.

[5] ATLAS Collaboration, Search for Higgs boson decays into pairs of light (pseudo)scalar particles in the $\gamma \gamma j j$ final state in pp collisions at $\sqrt{s}=13 \mathrm{TeV}$ with the ATLAS detector, Phys. Lett. B 782 (2018) 750, arXiv:1803.11145.

[6] ATLAS Collaboration, Identification and rejection of pile-up jets at high pseudorapidity with the ATLAS detector, Er. Phys. J. C 77 (2017) 580, arXiv:1705.02211. 